\title{
The influence of friction on the stress field of the Brazilian disc test: An experimental and theoretical analysis
}

\author{
Ch. F. Markides, D. N. Pazis, and S. K. Kourkoulis ${ }^{\text {a }}$ \\ National Technical University of Athens, School of Applied Mathematical and Physical Sciences, \\ Department of Mechanics, Zografou Campus, Theocaris Building, 5 Heroes of Polytechnion \\ Avenue, 15773 Athens, Greece
}

\begin{abstract}
A theoretical solution is proposed here for the determination of the stress field developed in an intact Brazilian disc in presence of tangential forces, based on the method of complex potentials introduced by Kolosov and Muskhelishvili. It is concluded that the influence of friction is negligible at the center of the disc and therefore it does not alter the value of the tensile strength obtained using the Brazilian disc test. On the contrary it is indicated that the changes induced on the stress field in the immediate vicinity of the rim are in favour of premature fracture very close at the ends of the rim, in accordance, also, to experimental findings.
\end{abstract}

\section{Introduction}

Determining the direct tensile strength of rock-type materials is a complicated experimental task. Serious difficulties have to be overcome related to the preparation of standardized specimens, the application of the load (due to the local fragmentation of the specimens) and the elimination of parasitic bending or torsional moments (due to even the slightest non co-axialities and misalignments). As a result, substitute tests were introduced, among which the Brazilian disc test [1] (i.e. the diametral compression of a compact cylinder) is the most popular one due to its simplicity.

The theoretical solution for the Brazilian disc was proposed by Hondros [2] for homogeneous, isotropic, linear elastic materials and uniform pressure distribution along two symmetric finite arcs of the periphery of the specimen. The scientific interest on the subject is continuous since then and finally ASTM established the specifications for the testing procedure and the specimen preparation [3] while the International Society of Rock Mechanics (ISRM) presented a "suggested" method for carrying out the experiment [4]. However and in spite of the intense research devoted to the subject a number of questions remain still unanswered. The influence of the friction forces generated at the disk-loading platen interface (due to the fact that the deformation properties of the disk and the platens are usually not identical) is among the most crucial ones. In a recent study Lavrov and Vervoort [5] concluded that although the stress field at the center of the disc does not depend on the friction along the loaded rim on the contrary the stress field in the vicinity of the platens is seriously affected.

In this direction an analytic solution of the problem is attempted here for the determination of the stress field in an intact Brazilian disk taking into account the friction forces inevitably developed at

\footnotetext{
a e-mail : stakkour@central.ntua.gr
} 
the loading platen-specimen interface. As a second step series of experiments were carried out with specimens made from either Dionysos marble or PMMA in order to check the validity of the results obtained from the theoretical analysis. In the second case (transparent specimens made from PMMA) the optical method of caustics was employed in an effort to quantify the influence of friction on the stress field developed in the immediate vicinity of the end-points of the load distribution.

\section{Theoretical considerations}

\subsection{The problem and the basic assumptions}

A closed form solution is obtained for the $1^{\text {st }}$ Fundamental Problem of Linear Elasticity for the intact Brazilian disc subjected to both a uniform radial compression as well as a uniform distribution of frictional tangential stresses along two symmetric arcs of its periphery (Figure 1). The direction of the frictional stresses, shown in Figure 1, is selected, by intuition, in the opposite sense from the direction along which the disc rim under the loading platens is expected to "move" due to the displacement field induced. The material of the disc is assumed to be linear elastic, isotropic and homogeneous. Treating the problem as a plane one the complex analysis is employed and specifically the method of complex potentials introduced by Kolosov and Muskhelishvili [6,7]. Advantage is taken of a recently introduced solution for the stress field in an intact Brazilian disc under exclusively uniform radial compression on two arcs of its periphery [8]. Therefore in the present study only the problem of the disc under frictional tangential stresses on its periphery is solved analytically. The final solution is obtained by superposition of the two stress fields (i.e. due to pressure and due to friction).

\subsection{The analytic solution and the results}

Consider a disc of radius $\mathrm{R}$ in the complex $\mathrm{z}$-plane, with $\mathrm{z}=\mathrm{x}+\mathrm{iy}$ or $z=r e^{i \vartheta}$. The origin of the Cartesian reference system is the center of the disc. An arbitrary point $\mathrm{z}$ on the disc's perimeter $\mathrm{L}$ is denoted by $t=\operatorname{Re}^{i \vartheta}$. Angle $\omega_{0}$, measured from the axis of sym-

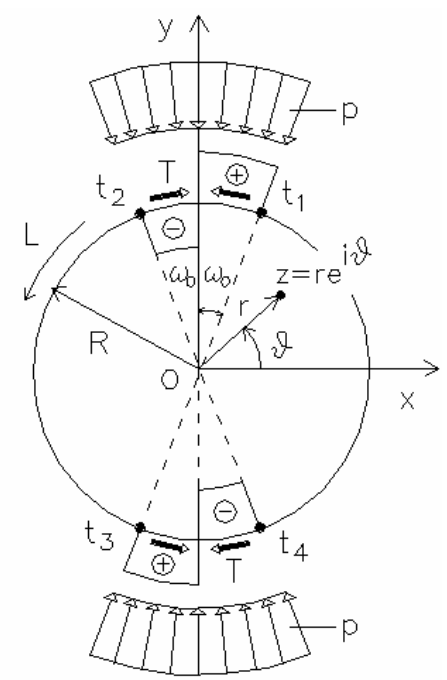

Fig. 1. The Brazilian disc under radial compression $\mathrm{p}$ and friction $\mathrm{T}$. metry of the external load, defines the extent of the distribution of the tangential stress $\mathrm{T}$. The end points of the load are denoted by $t_{j}, j=1,2,3,4$, in the counter clockwise sense. The problem is initially solved for the unit disc, in the fictitious complex $\zeta$-plane, with $\zeta=\xi+i \eta$ or $\zeta=\rho \mathrm{e}^{\mathrm{i} \vartheta}$. The points $t_{j}$ of $L$ correspond to the points $s_{j}, j=1,2,3$, 4 , on $\gamma$, where $s=e^{i \vartheta}$. The solution for the real disc is obtained using the trivial conformal transformation $\mathrm{z}=\omega(\zeta)=\mathrm{R} \zeta$ (Figure 2).

According to the method introduced by Muskhelishvili $[6,7]$ based on the properties of Cauchy integrals, the solution of the problem will be of the form:

$$
\varphi_{\mathrm{T}}(\zeta)=\frac{1}{2 \pi \mathrm{i}} \int_{\gamma} \frac{\mathrm{f}(\mathrm{s}) \mathrm{ds}}{\mathrm{s}-\zeta}-\overline{\mathrm{a}_{1}} \cdot \zeta, \quad \psi_{\mathrm{T}}(\zeta)=\frac{1}{2 \pi \mathrm{i}} \int_{\gamma} \frac{\overline{\mathrm{f}(\mathrm{s}) \mathrm{ds}}}{\mathrm{s}-\zeta}-\frac{\varphi_{\mathrm{T}}^{\prime}(\zeta)}{\zeta}+\frac{\mathrm{a}_{1}}{\zeta}
$$

where the index $\mathrm{T}$ denotes the tangential stress. The prime denotes the first order derivative. The overbar denotes the complex conjugate. For the complex potentials $\varphi_{\mathrm{T}}(\zeta)$ and $\psi_{\mathrm{T}}(\zeta)$ it holds that $\varphi_{\mathrm{T}}(\zeta)=$ $\int \Phi_{\mathrm{T}}(\zeta) \mathrm{d} \zeta$ and $\psi_{\mathrm{T}}(\zeta)=\int \Psi_{\mathrm{T}}(\zeta) \mathrm{d} \zeta$. The function $\mathrm{f}(\mathrm{s})$, giving the force acting on $\gamma$, is defined as:

$$
\mathrm{f}(\mathrm{s})=\mathrm{f}(\ell)=\mathrm{i} \int_{0}^{\ell}\left(\mathrm{X}_{\mathrm{T}}+\mathrm{i} \mathrm{Y}_{\mathrm{T}}\right) \mathrm{d} \ell
$$




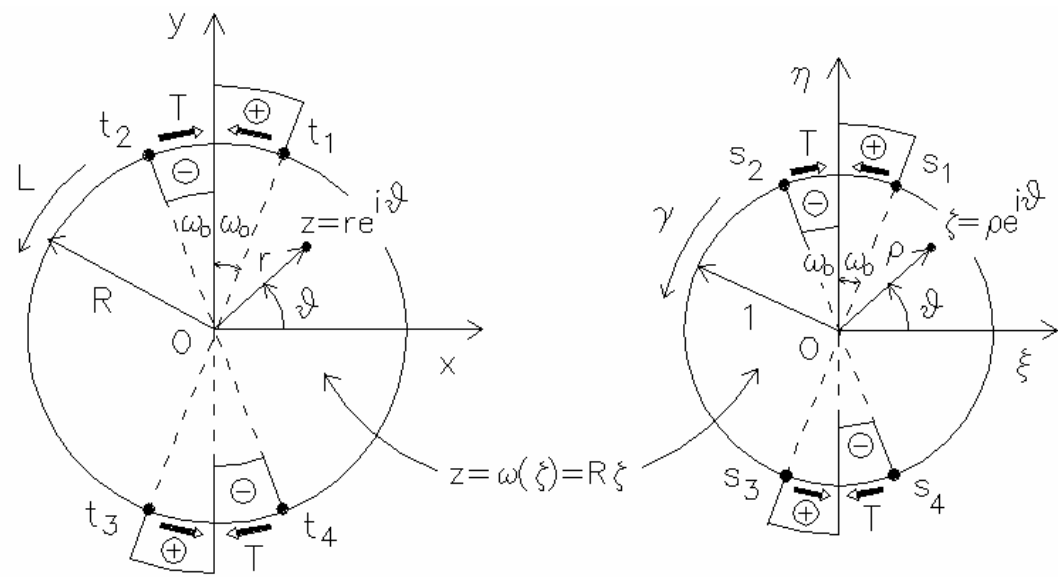

Fig. 2. Configuration of the mathematical problem, definitions of symbols and the conformal transformation.

with $\ell=1 \cdot \vartheta$ the length of the arc that corresponds to the point $s=e^{i \vartheta}$ on $\gamma$, measured in the counter clockwise direction from an arbitrarily fixed point $\mathrm{s}$ (e.g. the point $\mathrm{s}=1$ ). $\mathrm{X}_{\mathrm{T}}$ and $\mathrm{Y}_{\mathrm{T}}$, are the $\mathrm{X}^{-}, \mathrm{y}-$ components of the stress vector acting on $\gamma$, which for the present case are:

$$
\begin{array}{llr}
\mathrm{X}_{\mathrm{T}}=0, & \mathrm{Y}_{\mathrm{T}}=0 & \text { on the unloaded part of } \gamma, \\
\mathrm{X}_{\mathrm{T}}=-\mathrm{T} \sin \vartheta, & \mathrm{Y}_{\mathrm{T}}=\mathrm{T} \cos \vartheta & \pi / 2-\omega_{\mathrm{o}} \leq \vartheta<\pi / 2, \\
\mathrm{X}_{\mathrm{T}}=\mathrm{T} \sin \vartheta, & \mathrm{Y}_{\mathrm{T}}=-\mathrm{T} \cos \vartheta & \pi / 2<\vartheta \leq \pi / 2+\omega_{\mathrm{o}} \\
\mathrm{X}_{\mathrm{T}}=-\mathrm{T} \sin \vartheta, & \mathrm{Y}_{\mathrm{T}}=\mathrm{T} \cos \vartheta & 3 \pi / 2-\omega_{\mathrm{o}} \leq \vartheta<3 \pi / 2, \\
\mathrm{X}_{\mathrm{T}}=\mathrm{T} \sin \vartheta, & \mathrm{Y}_{\mathrm{T}}=-\mathrm{T} \cos \vartheta & 3 \pi / 2<\vartheta \leq 3 \pi / 2+\omega_{\mathrm{o}}
\end{array}
$$

Finally $\mathrm{a}_{1}$ is a constant defined as:

$$
\mathrm{a}_{1}=\overline{\mathrm{a}_{1}}=\frac{1}{4 \pi \mathrm{i}} \int_{\gamma} \frac{\mathrm{f}(\mathrm{s}) \mathrm{ds}}{\mathrm{s}^{2}}
$$

Introducing Eqs.(3) in Eq.( 2), one finds for the values of the function $\mathrm{f}(\mathrm{s})$ :

$$
\mathrm{f}(\mathrm{s})=\left\{\begin{array}{lll}
0 & \text { for } & 0 \leq \arg (\mathrm{s})<\pi / 2-\omega_{\mathrm{o}} \\
\mathrm{iT}\left(\mathrm{s}-\mathrm{s}_{1}\right) & \text { for } & \pi / 2-\omega_{\mathrm{o}} \leq \arg (\mathrm{s})<\pi / 2 \\
-2 \mathrm{~T}-\mathrm{iT}\left(\mathrm{s}+\mathrm{s}_{1}\right) & \text { for } & \pi / 2<\arg (\mathrm{s}) \leq \pi / 2+\omega_{\mathrm{o}} \\
-2 \mathrm{~T}-\mathrm{iT}\left(\mathrm{s}_{2}+\mathrm{s}_{1}\right) & \text { for } & \pi / 2+\omega_{\mathrm{o}}<\arg (\mathrm{s})<3 \pi / 2-\omega_{\mathrm{o}} \\
-2 \mathrm{~T}+\mathrm{iT}\left(\mathrm{s}-\mathrm{s}_{2}\right) & \text { for } & 3 \pi / 2-\omega_{\mathrm{o}} \leq \arg (\mathrm{s})<3 \pi / 2 \\
-\mathrm{iT}\left(\mathrm{s}+\mathrm{s}_{2}\right) & \text { for } & 3 \pi / 2<\arg (\mathrm{s}) \leq 3 \pi / 2+\omega_{\mathrm{o}} \\
0 & \text { for } & 3 \pi / 2+\omega_{\mathrm{o}}<\arg (\mathrm{s})<0
\end{array}\right.
$$

Substitution of $\mathrm{f}(\mathrm{s})$ from Eqs.(5), in Eq.(1) and Eq.(4), yields $\varphi_{\mathrm{T}}(\zeta)$ and $\psi_{\mathrm{T}}(\zeta)$ as:

$$
\begin{aligned}
& \psi_{\mathrm{T}}(\zeta)=\frac{\mathrm{T}}{\pi}\left[\frac{\mathrm{s}_{2}}{2} \cdot \ln \frac{\mathrm{s}_{1}-\zeta}{\mathrm{s}_{1}+\zeta}+\frac{\mathrm{s}_{1}}{2} \cdot \ln \frac{\mathrm{s}_{2}-\zeta}{\mathrm{s}_{2}+\zeta}-\mathrm{i} \cdot \ln \frac{\mathrm{i}-\zeta}{\mathrm{i}+\zeta}-\frac{2 \pi \mathrm{i}}{\zeta}-\frac{2}{\zeta} \cdot \ln \left(1+\zeta^{2}\right)+\right. \\
& \frac{1}{\zeta} \cdot \ln \left(\mathrm{s}_{1}{ }^{2}-\zeta^{2}\right)\left(\mathrm{s}_{2}{ }^{2}-\zeta^{2}\right)+\frac{\mathrm{s}_{1}}{2 \zeta}\left(\frac{1}{\mathrm{~s}_{1}-\zeta}+\frac{1}{\mathrm{~s}_{1}+\zeta}\right)+\frac{\mathrm{s}_{2}}{2 \zeta}\left(\frac{1}{\mathrm{~s}_{2}-\zeta}+\frac{1}{\mathrm{~s}_{2}+\zeta}\right)-\frac{\mathrm{i}}{\zeta}\left(\frac{1}{\mathrm{i}-\zeta}+\frac{1}{\mathrm{i}+\zeta}\right)- \\
& \left.\frac{2 \zeta}{1+\zeta^{2}}-\zeta \cdot\left(\frac{1}{\mathrm{~s}_{1}{ }^{2}-\zeta^{2}}+\frac{1}{\mathrm{~s}_{2}{ }^{2}-\zeta^{2}}\right)-\pi \cdot\left(1-\cos \omega_{\mathrm{o}}\right)\right]
\end{aligned}
$$




$$
\begin{aligned}
& \varphi_{\mathrm{T}}(\zeta)=\frac{\mathrm{T}}{\pi}\left[\frac{\mathrm{s}_{1}}{2} \cdot \ln \frac{\mathrm{s}_{1}-\zeta}{\mathrm{s}_{1}+\zeta}+\frac{\mathrm{s}_{2}}{2} \cdot \ln \frac{\mathrm{s}_{2}-\zeta}{\mathrm{s}_{2}+\zeta}-\mathrm{i} \cdot \ln \frac{\mathrm{i}-\zeta}{\mathrm{i}+\zeta}+\pi \mathrm{i} \zeta+\zeta \cdot \ln \left(1+\zeta^{2}\right)-\right. \\
& \left.\frac{\zeta}{2} \cdot \ln \left(\mathrm{s}_{1}{ }^{2}-\zeta^{2}\right)\left(\mathrm{s}_{2}{ }^{2}-\zeta^{2}\right)-\pi \cdot\left(1-\cos \omega_{\mathrm{o}}\right)\right]
\end{aligned}
$$

Differentiating now the last expressions and using the conformal transformation $\mathrm{z}=\omega(\zeta)=\mathrm{R} \zeta$ one obtains the solution for the disc of radius $\mathrm{R}$ as follows:

$$
\begin{aligned}
& \Psi_{\mathrm{T}}(\mathrm{z})=\frac{\mathrm{T}}{\pi}\left\{-\frac{\mathrm{t}_{1}}{\mathrm{t}_{1}^{2}-\mathrm{z}^{2}}\left(\mathrm{t}_{2}+\frac{\mathrm{t}_{1} \mathrm{R}^{2}}{\mathrm{z}^{2}}\right)-\frac{\mathrm{t}_{2}}{\mathrm{t}_{2}^{2}-\mathrm{z}^{2}}\left(\mathrm{t}_{1}+\frac{\mathrm{t}_{2} \mathrm{R}^{2}}{\mathrm{z}^{2}}\right)+\frac{2 \mathrm{R}^{4}}{\mathrm{z}^{2}\left(\mathrm{R}^{2}+\mathrm{z}^{2}\right)}+\right. \\
& \frac{2 \mathrm{R}^{2}}{\mathrm{z}^{2}}\left[\pi \mathrm{i}+\ln \left(\mathrm{R}^{2}+\mathrm{z}^{2}\right)-\frac{1}{2} \ln \left(\mathrm{t}_{1}^{2}-\mathrm{z}^{2}\right)\left(\mathrm{t}_{2}^{2}-\mathrm{z}^{2}\right)\right]-3 \mathrm{R}^{2}\left(\frac{1}{\mathrm{t}_{1}^{2}-\mathrm{z}^{2}}+\frac{1}{\mathrm{t}_{2}{ }^{2}-\mathrm{z}^{2}}\right)+ \\
& \left.2 \mathrm{R}^{2}\left[\frac{\mathrm{t}_{1}{ }^{2}}{\left(\mathrm{t}_{1}{ }^{2}-\mathrm{z}^{2}\right)^{2}}+\frac{\mathrm{t}_{2}{ }^{2}}{\left(\mathrm{t}_{2}{ }^{2}-\mathrm{z}^{2}\right)^{2}}\right]-2 \mathrm{z}^{2} \mathrm{R}^{2}\left[\frac{1}{\left(\mathrm{t}_{1}{ }^{2}-\mathrm{z}^{2}\right)^{2}}+\frac{1}{\left(\mathrm{t}_{2}{ }^{2}-\mathrm{z}^{2}\right)^{2}}\right]\right\} \\
& \Phi_{\mathrm{T}}(\mathrm{z})=\frac{\mathrm{T}}{\pi}\left[2-\left(\frac{\mathrm{t}_{1}^{2}}{\mathrm{t}_{1}{ }^{2}-\mathrm{z}^{2}}+\frac{\mathrm{t}_{2}^{2}}{\mathrm{t}_{2}{ }^{2}-\mathrm{z}^{2}}\right)+\pi \mathrm{i}+\ln \left(\mathrm{R}^{2}+\mathrm{z}^{2}\right)-\right. \\
& \left.\frac{1}{2} \ln \left(\mathrm{t}_{1}^{2}-\mathrm{z}^{2}\right)\left(\mathrm{t}_{2}^{2}-\mathrm{z}^{2}\right)+\mathrm{z}^{2} \cdot\left(\frac{1}{\mathrm{t}_{1}^{2}-\mathrm{z}^{2}}+\frac{1}{\mathrm{t}_{2}^{2}-\mathrm{z}^{2}}\right)\right]
\end{aligned}
$$

Introduction of Eqs. $(8,9)$, in the polar expressions for the stresses [7]:

$$
\sigma_{\mathrm{rr}}^{\mathrm{T}}+\sigma_{\vartheta \vartheta}^{\mathrm{T}}=4 \Re \Phi_{\mathrm{T}}(\mathrm{z}), \quad \sigma_{\mathrm{rr}}^{\mathrm{T}}-\mathrm{i} \sigma_{\mathrm{r} \vartheta}^{\mathrm{T}}=2 \Re \Phi_{\mathrm{T}}(\mathrm{z})-\mathrm{e}^{2 \mathrm{i} \vartheta}\left[\overline{\mathrm{z}} \cdot \Phi_{\mathrm{T}}^{\prime}(\mathrm{z})+\Psi_{\mathrm{T}}(\mathrm{z})\right]
$$

yields the stress components due to the friction forces in closed form for any point of the disc as:

$$
\begin{aligned}
& \underset{\vartheta \vartheta}{\sigma_{\mathrm{rr}}^{\mathrm{T}}}=\frac{2 \mathrm{~T}}{\pi}\left\{( 1 \mp \frac { \mathrm { R } ^ { 2 } } { \mathrm { r } ^ { 2 } } ) \cdot \left[\ln \sqrt{\mathrm{R}^{4}+2 \mathrm{r}^{2} \mathrm{R}^{2} \cos 2 \vartheta+\mathrm{r}^{4}}-\frac{1}{2}\left(\ell n \sqrt{\mathrm{R}^{4}+2 \mathrm{r}^{2} \mathrm{R}^{2} \cos 2\left(\vartheta+\omega_{\mathrm{o}}\right)+\mathrm{r}^{4}}+\right.\right.\right. \\
& \left.\left.\ell n \sqrt{R^{4}+2 r^{2} R^{2} \cos 2\left(\vartheta-\omega_{0}\right)+r^{4}}\right)\right] \mp \frac{R^{6}+r^{2} R^{2}\left(R^{2}+r^{2}\right) \cos 2 \vartheta+r^{6}}{r^{2}\left(R^{4}+2 r^{2} R^{2} \cos 2 \vartheta+r^{4}\right)} \pm \\
& \frac{\left(\mathrm{R}^{2}-\mathrm{r}^{2}\right)\left[\mathrm{R}^{2}\left(\mathrm{R}^{4}+3 \mathrm{r}^{4}\right) \cos 2\left(\vartheta+\omega_{\mathrm{o}}\right)+\mathrm{r}^{2}\left(\mathrm{r}^{4}+\mathrm{R}^{4}\left(2+\cos 4\left(\vartheta+\omega_{\mathrm{o}}\right)\right)\right)\right]}{\left[\mathrm{R}^{4}+2 \mathrm{r}^{2} \mathrm{R}^{2} \cos 2\left(\vartheta+\omega_{\mathrm{o}}\right)+\mathrm{r}^{4}\right]^{2}} \pm \\
& \frac{\left(\mathrm{R}^{2}-\mathrm{r}^{2}\right)\left[\mathrm{R}^{2}\left(\mathrm{R}^{4}+3 \mathrm{r}^{4}\right) \cos 2\left(\vartheta-\omega_{\mathrm{o}}\right)+\mathrm{r}^{2}\left(\mathrm{r}^{4}+\mathrm{R}^{4}\left(2+\cos 4\left(\vartheta-\omega_{\mathrm{o}}\right)\right)\right)\right]}{\left[\mathrm{R}^{4}+2 \mathrm{r}^{2} \mathrm{R}^{2} \cos 2\left(\vartheta-\omega_{\mathrm{o}}\right)+\mathrm{r}^{4}\right]^{2}} \pm \\
& \frac{3 r^{2}-2 R^{2}}{2} \cdot\left[\frac{r^{2}+R^{2} \cos 2\left(\vartheta+\omega_{o}\right)}{R^{4}+2 r^{2} R^{2} \cos 2\left(\vartheta+\omega_{0}\right)+r^{4}}+\frac{r^{2}+R^{2} \cos 2\left(\vartheta-\omega_{o}\right)}{R^{4}+2 r^{2} R^{2} \cos 2\left(\vartheta-\omega_{o}\right)+r^{4}}\right] \pm \\
& \left.\frac{R^{4}}{2 r^{2}} \cdot\left[\frac{R^{2}+r^{2} \cos 2\left(\vartheta+\omega_{o}\right)}{R^{4}+2 r^{2} R^{2} \cos 2\left(\vartheta+\omega_{o}\right)+r^{4}}+\frac{R^{2}+r^{2} \cos 2\left(\vartheta-\omega_{o}\right)}{R^{4}+2 r^{2} R^{2} \cos 2\left(\vartheta-\omega_{o}\right)+r^{4}}\right]\right\}
\end{aligned}
$$




$$
\begin{aligned}
& \sigma_{\mathrm{r} \vartheta}^{\mathrm{T}}=\frac{2 \mathrm{~T}}{\pi} \cdot \mathrm{R}^{2} \cdot\left\{( \mathrm { R } ^ { 2 } - \mathrm { r } ^ { 2 } ) \cdot \left[\frac{\sin 2\left(\vartheta+\omega_{\mathrm{o}}\right)}{2\left(\mathrm{R}^{4}+2 \mathrm{r}^{2} \mathrm{R}^{2} \cos 2\left(\vartheta+\omega_{\mathrm{o}}\right)+\mathrm{r}^{4}\right)}+\right.\right. \\
& \left.\frac{\sin 2\left(\vartheta-\omega_{\mathrm{o}}\right)}{2\left(\mathrm{R}^{4}+2 \mathrm{r}^{2} \mathrm{R}^{2} \cos 2\left(\vartheta-\omega_{\mathrm{o}}\right)+\mathrm{r}^{4}\right)}-\frac{\sin 2 \vartheta}{\mathrm{R}^{4}+2 \mathrm{r}^{2} \mathrm{R}^{2} \cos 2 \vartheta+\mathrm{r}^{4}}\right]+\frac{1}{\mathrm{r}^{2}}\left[\operatorname{Arctan} \frac{\mathrm{r}^{2} \sin 2 \vartheta}{\mathrm{R}^{2}+\mathrm{r}^{2} \cos 2 \vartheta}+\right. \\
& \left.\left.\frac{1}{2} \cdot\left(\operatorname{Arctan} \frac{\mathrm{R}^{2} \sin 2 \omega_{\mathrm{o}}-\mathrm{r}^{2} \sin 2 \vartheta}{\mathrm{R}^{2} \cos 2 \omega_{\mathrm{o}}+\mathrm{r}^{2} \cos 2 \vartheta}-\operatorname{Arctan} \frac{\mathrm{R}^{2} \sin 2 \omega_{\mathrm{o}}+\mathrm{r}^{2} \sin 2 \vartheta}{\mathrm{R}^{2} \cos 2 \omega_{\mathrm{o}}+\mathrm{r}^{2} \cos 2 \vartheta}\right)\right]\right\}
\end{aligned}
$$

In these expressions the $(+)$ sign corresponds to $\sigma_{\mathrm{rr}}^{\mathrm{T}}$ while the $(-)$ sign corresponds to $\sigma_{\vartheta \vartheta}^{\mathrm{T}}$.

It can be readily seen from Eqs. $(11,12)$ that the distribution of friction stresses assumed here does not influence the center of the disc. Indeed the stress components vanish in the center of the disc, as it was to be expected due to the antisymmetry of the problem. On the contrary a strong gradient of the stress field at the end points of the loaded rim can be detected. Namely the normal stress $\sigma_{\vartheta \vartheta}^{\mathrm{T}}$ becomes infinite at the point $t_{1}$ :

$$
\left.\lim _{r \rightarrow R}\right|_{\vartheta=\frac{\pi}{2}-\omega_{0}} \sigma_{\vartheta \vartheta}^{\mathrm{T}}=\left.\lim _{\mathrm{r} \rightarrow \mathrm{R}}\right|_{\vartheta=\frac{\pi}{2}-\omega_{0}} \frac{2 \mathrm{~T}}{\pi} \cdot\left\{\left(1+\frac{\mathrm{R}^{2}}{\mathrm{r}^{2}}\right) \cdot\left[\ldots-\frac{1}{2} \ln \left(\mathrm{R}^{2}-\mathrm{r}^{2}\right)-\ldots\right] \ldots\right\}=-(-\infty)=+\infty
$$

This fact in conjunction to the changing of the sign of $\sigma_{\vartheta \vartheta}^{\mathrm{T}}$ from both sides of the point $\mathrm{t}_{1}$ [being positive on the unloaded part of $\mathrm{L}\left(0 \leq \vartheta<90-\omega_{0}\right)$ and negative on the loaded rim $\left.\left(90-\omega_{0}<\vartheta \leq 90\right)\right]$, shows a tendency of failure of the disc at the end points of the loading.

The solution of the overall problem, with friction $\mathrm{T}$ and radial compression $\mathrm{p}$, follows directly by the superposition of the solution just obtained for the friction field to the one obtained in [8] for the radial pressure, considering simply $\mathrm{T}$ as a Coulomb friction stress given as $\mathrm{T}=f \mathrm{p}$ ( $f$ is the coefficient of friction along the loaded rim).

Applying the above formulae the stress field components developed are plotted in Figure 3, along three characteristic directions, i.e. the diameter normal to the load (x-axis), the diameter parallel to the load (y-axis) and the diameter along the end-point of the external load distributions $\left(\vartheta=85^{\circ}\right)$. The stress field for each case due to the radial pressure [Figures $3(\mathrm{a} 1, \mathrm{~b} 1, \mathrm{c} 1)$ ] is plotted in juxtaposition to the respective field due to the friction forces [Figures $3(\mathrm{a} 2, \mathrm{~b} 2, \mathrm{c} 2)$ ]. It is obvious from these figures that friction is only worth dealing with in the immediate vicinity of the loaded rim. Indeed along the $\mathrm{x}$-axis the perturbation of the stress field due to friction is almost zero. For example the $\sigma_{\vartheta \vartheta}$ stress component due to $\mathrm{p}$ is $10^{4}$ times higher from the respective one due to friction.

The situation is dramatically different along y-axis. It is observed from Figures $3(\mathrm{~b} 1, \mathrm{~b} 2)$ that as the loaded rim is approached $(r \rightarrow R)$ the stress components due to friction obtain values well comparable to those due to pressure. In fact for $r>0.48 \mathrm{R}$ the stresses due to $\mathrm{T}$ exceed significantly those due to $\mathrm{p}$. From a quantitative point of view the influence of friction along the line $\vartheta=85^{\circ}$ is of the same nature. What is interesting to note is the sign change of the $\sigma_{\vartheta \vartheta}$ stress component due to friction, which is now of tensile nature, counterbalancing the influence of the respective component due to pressure.

\section{Experimental study}

In an effort to quantify experimentally the changes induced on the stress field due to friction series of Brazilian disc tests were carried out using specimens made from Dionysos marble, the material used in the restoration project of the Parthenon Temple of Athens. It behaves almost linearly elastic for a significant portion of its axial stress - axial strain curve permitting direct comparison of the experimental data with the respective ones of the theoretical study of the previous paragraph. 
EPJ Web of Conferences
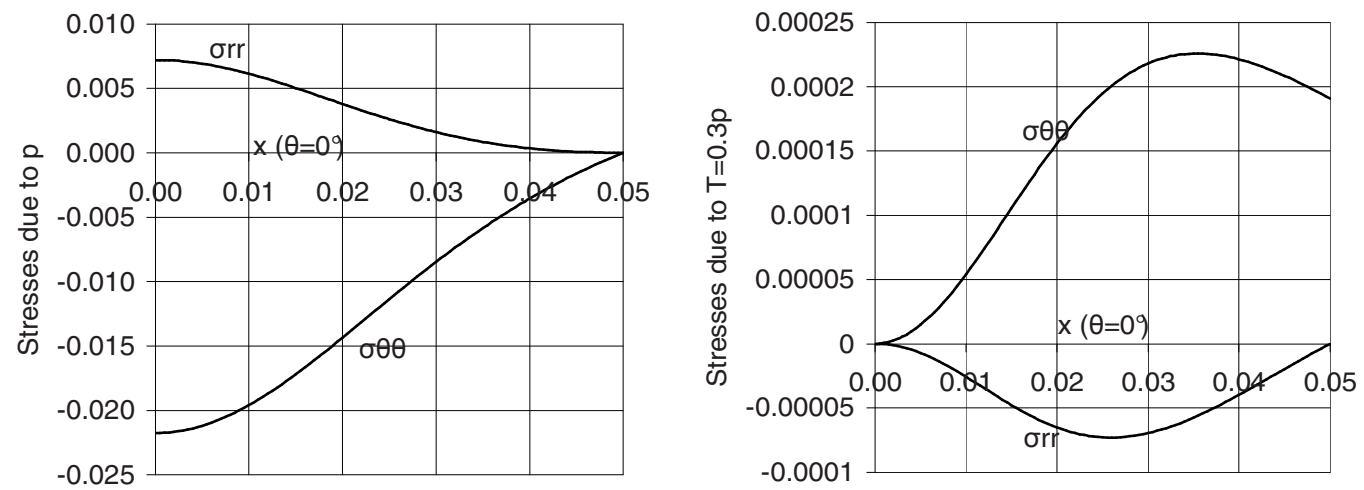

(a1)

(a2)
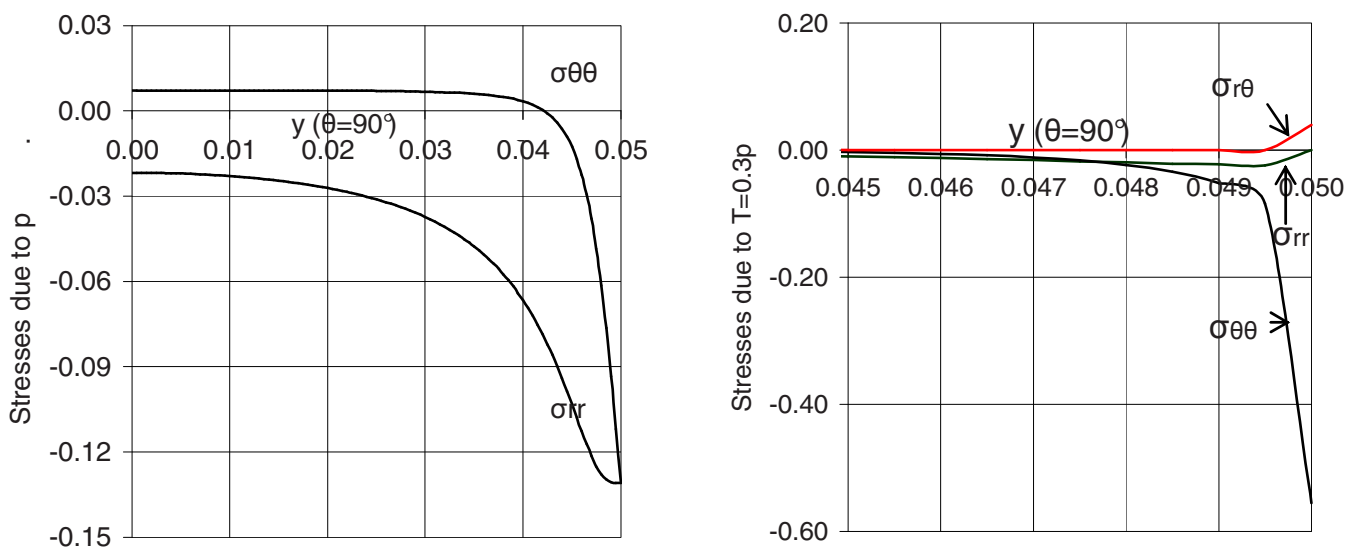

(b1)

(b2)
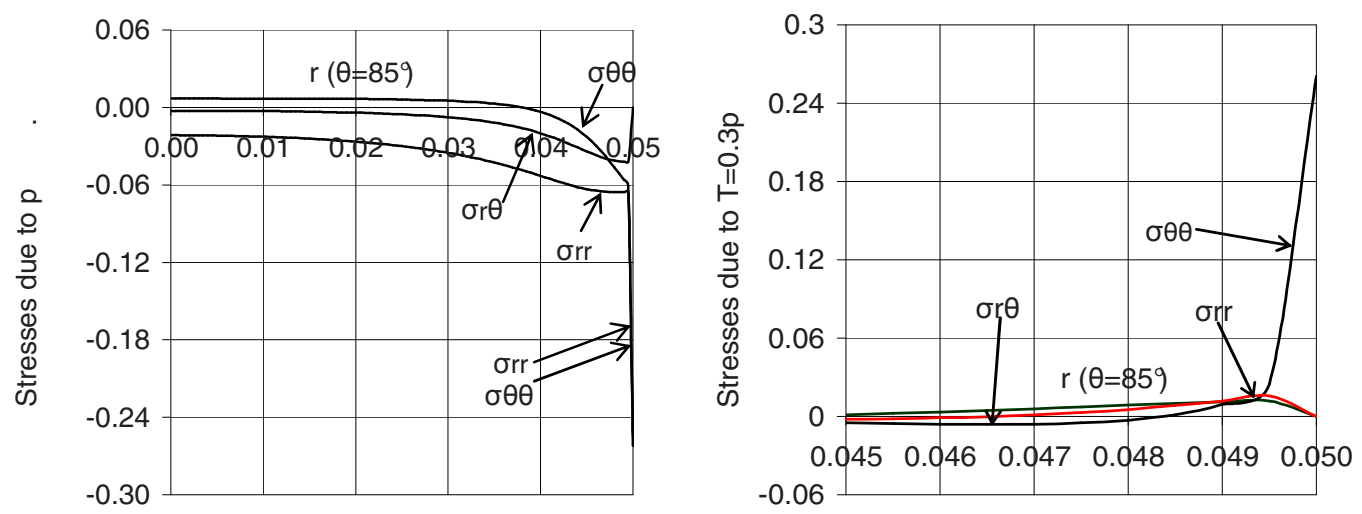

(c1)

(c2)

Fig. 3. The stress components variations along the:

(a) $\mathrm{x}$-axis, i.e. the radius normal to the load axis, due to $\mathrm{p}$ (a1) and due to $\mathrm{T}$ (a2)

(b) y-axis, i.e. the radius along the load axis, due to $\mathrm{p}$ (b1) and due to $\mathrm{T}$ (b2)

(c) radius with $\theta=85^{\circ}$, i.e. towards the end-point of the load distribution, due to $\mathrm{p}(\mathrm{c} 1)$ and due to $\mathrm{T}(\mathrm{c} 2)$. 
The specimens were disc-shaped (diameter $100 \mathrm{~mm}$, thickness $50 \mathrm{~mm}$ ). The slightly transversely isotropic nature of Dionysos marble was ignored [9]. All specimens were loaded using the device suggested by ISRM (Figure 4). The load was applied by a stiff hydraulic frame of capacity $1 \mathrm{MN}$. The loading rate was very low resulting to a displacement rate not exceeding $0.02 \mathrm{~mm} / \mathrm{min}$.

The friction conditions were controlled by interposing inserts of various relative roughnesses with respect to the marble. The strains developed were measured using strain gauge rosettes (Figure 4). From the strain field components the stress field was ob-

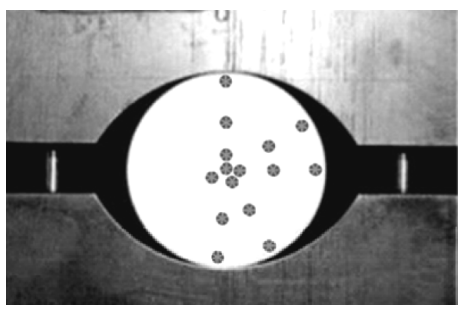

Fig. 4. The experimental set-up and the arrangement of strain gauges. tained adopting Hooke's generalized law.

The experimental results indicated indeed that the stress field at the center of the disc is insensitive to the changes of the coefficient of friction, at least for the values tested (ranging from about 0.2 to almost 1.0). In fact the variations of the stress field recorded by the central rosette (Figure 4) were statistically insignificant. On the contrary the stress field in the vicinity of the loaded rim was drastically altered influencing even the failure mode: Instead of fracture starting from the center of the disc cracks appeared starting from the load end-points. The tests were repeated with specimens made from a softer stone, from Crete Island (Figure $5)$, since it was easier to obtain higher coefficients of friction. The conclusions were of the same nature: Increased friction causes premature failure in the vicinity of the load-platens.

Finally a small number of tests were carried out using specimens made from transparent material in order to study the stress field in the vicinity of the loaded point using the optical method of Caustics (for practical reasons gauges cannot be attached very close to the platen-specimen contact point). The

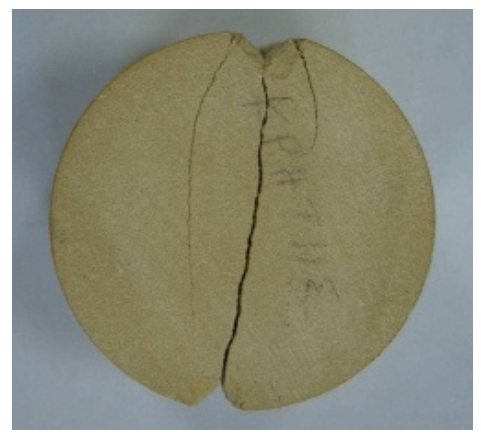

Fig. 5. Fractured specimen from Alfopetra stone (from Crete). optical method of Caustics is a powerful experimental technique due to its high sensitivity to stress gradients. It was Manogg [10] in 1964 who introduced the method of transmitted caustics and Theocaris [11] in 1970 who introduced the method of reflected caustics. A typical arrangement used for realization of the method is shown in Figure 6.

The material used was the optically isotropic Poly-methyl-meth-acrylate (PMMA) known under the commercial name plexiglas. Preliminary standardized tests were carried out in order to determine the mechanical properties of the specific batch of the material. The values of the mechanical constants finally adopted from these preliminary tests were $\mathrm{E}=3.3 \mathrm{GPa}, v=0.38$ and the yield stress was estimated to about $28 \mathrm{MPa}$. Having defined the mechanical properties one can determine the stress-

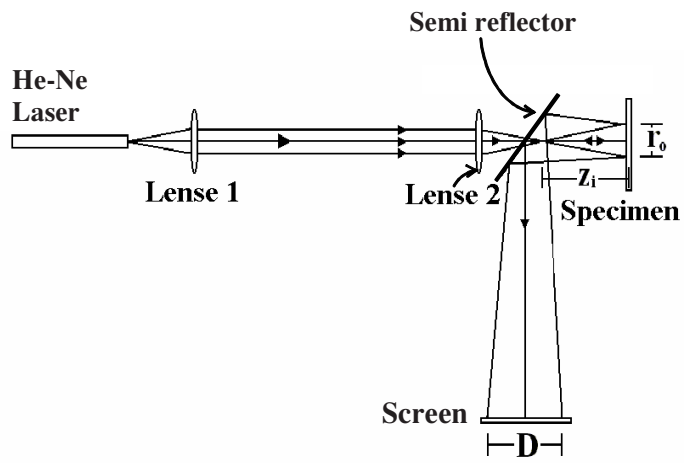

Fig. 6. The optical set-up for the method of Caustics with a divergent light beam. optical constants of PMMA which were found equal to: $\mathrm{c}_{\mathrm{f}}=1.14 \times 10^{-5} \mathrm{~cm}^{2} / \mathrm{kp}$ and $\mathrm{c}_{\mathrm{r}}=-1.82 \times 10^{-5}$ $\mathrm{cm}^{2} / \mathrm{kp}$. A characteristic series of photos exhibiting the evolution of caustics developed due to the contact of the load platen and the PMMA specimen during a typical test is shown in Figure 7. Figure 7a corresponds to the unloaded specimen (the caustic formed is due to the superimposed weight if the ISRM device) while Figure $7 \mathrm{~d}$ corresponds to a load just before the fracture of the specimen. Although the theoretical analysis of the caustics shape, due to contact, is at a preliminary stage, the comparison of the present experimental results with those of earlier approaches by Theocaris and Stassinakis $[12,13]$ 


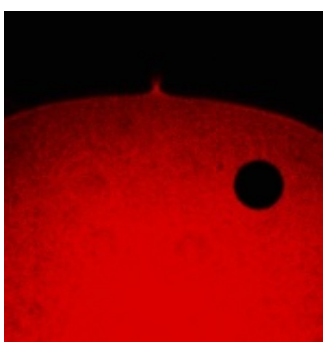

(a)

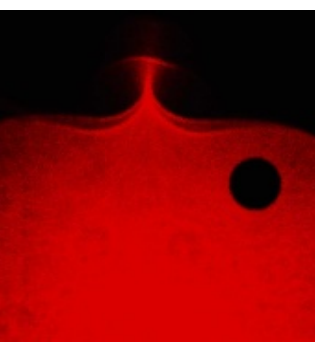

(b)

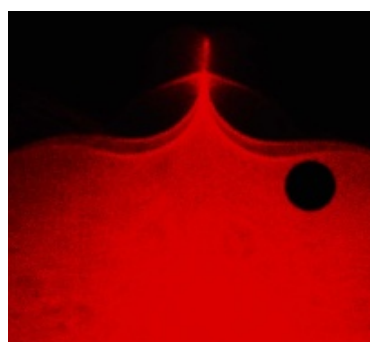

(c)

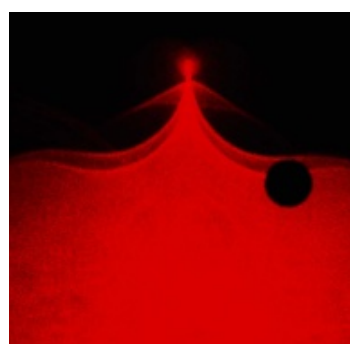

(d)

Fig. 7: The evolution of the contact caustics from the unloaded state (a) up to a load level just before fracture (d) for a coefficient of friction equal to 0.3. (The black marker is one Euro coin)

are encouraging, indicating that the stress field amplification due to friction are in good agreement with the results of the present analysis.

\section{Conclusions}

A theoretical solution for the stress field in an intact Brazilian disc was obtained considering the influence of the friction forces developed at the loading platen - specimen interface. It was concluded that the value of the Brazilian test is not deteriorated due to the friction itself since the stress field in the center of the disc is almost absolutely insensitive to the friction effects. However what is to be seriously considered is that in some cases the presence of friction may cause premature failure near the loading platens due to the local amplification of the stress field. The experimental investigation of the problem using marble specimens verified the main conclusion, with the aid of point measurements obtained from strain gauge rosettes. Since for practical reasons the gauges cannot give results from points close enough to the contact point between the platen and the specimen, the method of caustics was employed. Preliminary results indicate a rather satisfactory agreement using the theoretical shape of caustics due to contact obtained from earlier studies.

\section{References}

1. F. Carneiro, A. Barcellos, Bulletin Rilem, I(13), 97-108 (1953)

2. G. Hondros, Australian Journal of Applied Science, 10, 243-268 (1959)

3. ASTM, Standard Method for Splitting Tensile Strength of Intact Rock Core Specimens, 04.08, 471-475 (1988)

4. ISRM (F. Ouchterlony, Co-ordinator), Int. J. Rock Mech. Min. Sci. Geomech. Abstr., 25, 71-96 (1988)

5. A. Lavrov, A. Vervoort, Int. J. Rock Mech. Mining Sci., 39(2), 275-283 (2002)

6. N. I. Muskhelishvili, Some Basic Problems of the Mathematical Theory of Elasticity (P. Noordhoff, Groningen, The Netherlands, 1963)

7. N. I. Muskhelishvili, Singular Integral Equations (Wolters - Noordhoff, Groningen, The Netherlands, 1958)

8. Ch. F.Markides, D. N. Pazis, S. K. Kourkoulis, Int. J. Rock Mech. Min. Sci, 47, 227-237 (2010)

9. G. E. Exadaktylos, I. Vardoulakis, S. K. Kourkoulis, International Journal of Solids and Structures, 38, 4119-4145 (2001)

10. P. Manogg, Anwendung der Schattenoptik zur Untersuchung des Zerreissvongangs von Platten. Dissertation, Freiburg, Germany, (1964)

11. P. S. Theocaris, Local yielding around a crack tip in Plexiglas. Journal of Applied Mechanics, 37, 409-415, (1970)

12. P. S. Theocaris, C. A. Stassinakis, A., Experimental Mechanics, 18, 409-415 (1978)

13. P. S. Theocaris, C. A. Stassinakis, J. Eng. Mater. Technol., 106(3) (1984) 